\title{
In Vivo Carbon-13 Dynamic MRS and MRSI of Normal and Fasted Rat Liver with Hyperpolarized ${ }^{13} \mathrm{C}$-Pyruvate
}

Simon Hu, ${ }^{1,2}$ Albert P. Chen, ${ }^{3}$ Matthew L. Zierhut, ${ }^{1,2}$ Robert Bok, ${ }^{1}$ Yi-Fen Yen, ${ }^{3}$ Marie A. Schroeder, ${ }^{4}$ Ralph E. Hurd, ${ }^{3}$ Sarah J. Nelson, ${ }^{1,2}$ John Kurhanewicz, ${ }^{1,2}$ Daniel B. Vigneron ${ }^{1,2}$

${ }^{1}$ Department of Radiology and Biomedical Imaging, University of California, San Francisco, Box 251217004 th St. QB3 Building, Suite 102, San Francisco, CA 94158-2512, USA

${ }^{2} U C S F$ \& UCB Joint Graduate Group in Bioengineering, Berkeley, CA, USA

${ }^{3}$ GE Healthcare, Menlo Park, CA, USA

${ }^{4}$ Department of Physiology, University of Oxford, Oxford, UK

\begin{abstract}
Background: The use of in vivo ${ }^{13} \mathrm{C}$ nuclear magnetic resonance spectroscopy in probing metabolic pathways to study normal metabolism and characterize disease physiology has been limited by its low sensitivity. However, recent technological advances have enabled greater than 50,000 -fold enhancement of liquid-state polarization of metabolically active ${ }^{13} \mathrm{C}$ substrates, allowing for rapid assessment of ${ }^{13} \mathrm{C}$ metabolism in vivo. The present study applied hyperpolarized ${ }^{13} \mathrm{C}$ magnetic resonance spectroscopy to the investigation of liver metabolism, demonstrating for the first time the feasibility of applying this technology to detect differences in liver metabolic states.

Procedures: $\left[1-{ }^{13} \mathrm{C}\right]$ pyruvate was hyperpolarized with a dynamic nuclear polarization instrument and injected into normal and fasted rats. The uptake of pyruvate and its conversion to the metabolic products lactate and alanine were observed with slice-localized dynamic magnetic resonance spectroscopy and 3D magnetic resonance spectroscopic imaging (3D-MRSI).

Results: Significant differences in lactate to alanine ratio $(P<0.01)$ between normal and fasted rat liver slice dynamic spectra were observed. 3D-MRSI localized to the fasted livers demonstrated significantly decreased ${ }^{13} \mathrm{C}$-alanine levels $(P<0.01)$ compared to normal.

Conclusions: This study presents the initial demonstration of characterizing metabolic state differences in the liver with hyperpolarized ${ }^{13} \mathrm{C}$ spectroscopy and shows the ability to detect physiological perturbations in alanine aminotransferase activity, which is an encouraging result for future liver disease investigations with hyperpolarized magnetic resonance technology.
\end{abstract}

Key words: Hyperpolarized carbon-13, Dynamic nuclear polarization (DNP), Magnetic resonance spectroscopy/spectroscopic imaging, Fasted liver, Alanine aminotransferase 


\section{Introduction}

I mproved monitoring and measurement of metabolism in key biological processes, such as glycolysis and the citric acid cycle, both in the presence and absence of disease, have been long-sought goals in the molecular and medical imaging communities. In particular, there has been great interest in pursuing carbon-13 nuclear magnetic resonance spectroscopy as a means of probing metabolic pathways to study normal metabolism and characterize disease physiology [1-3]. While these in vivo studies of either endogenous or enriched ${ }^{13} \mathrm{C}$ compounds have proven fruitful, such experiments have suffered from unique technical challenges, the most severe of which is low signal sensitivity, resulting in long acquisition times and large voxel sizes to obtain spectra with adequate signal-to-noise ratios (SNR) [1-3]. However, a recent technological breakthrough, namely the development of techniques to maintain hyperpolarization of carbon-13 substrates in liquid state [4-5], has generated significant excitement for the prospect of rapid high signalto-noise measurement of ${ }^{13} \mathrm{C}$ metabolism in vivo. Hyperpolarization [4-5] was achieved through the dynamic nuclear polarization (DNP) effect and thermal cooling, and liquidstate (compatible with in vivo injection) polarization in excess of 50,000-fold was preserved using a novel rapid heated dissolution process. The use of carbon-13-enriched substrates as molecular imaging agents with this technique is also attractive because ${ }^{13} \mathrm{C}$ is not radioactive and is only slightly heavier than carbon endogenous to the body, causing no significant isotope effects.
Initial applications of this technology were primarily focused on tracer-based angiography [6] and perfusion mapping [7]. More recent studies have included metabolic molecular imaging, using the metabolically active substrate $\left[1-{ }^{13} \mathrm{C}\right]$ pyruvate to examine its conversion to $\left[1-{ }^{13} \mathrm{C}\right]$ lactate, $\left[1-{ }^{13} \mathrm{C}\right]$ alanine, and ${ }^{13} \mathrm{C}$-bicarbonate [8-12]. Fig. 1 provides a simplified summary of the metabolic pathways studied in vivo with hyperpolarized $\left[1-{ }^{13} \mathrm{C}\right]$ pyruvate. (Note: $\mathrm{LDH}$ can also be found in the mitochondria [13].) This hyperpolarized metabolic imaging approach enables not only the detection of the uptake of the injected prepolarized substrate such as pyruvate but also its conversion to lactate or alanine due to rapid exchange of the ${ }^{13} \mathrm{C}$ label [12] as catalyzed by lactate dehydrogenase (LDH) and alanine aminotransferase (ALT), respectively. In prior studies, significantly higher levels of $\left[1-{ }^{13} \mathrm{C}\right]$ lactate were found in implanted mouse tumors [9, 12] and tumors in a mouse model of prostate cancer [11], which can be explained by the hyperglycolytic state and increased LDH activity common to most cancers [14-15]. Furthermore, it was demonstrated that the rate constant for $\mathrm{LDH}$ flux decreased after drug treatment in an implanted tumor model [12]. Thus, using hyperpolarized magnetic resonance imaging (MRI) technology to monitor LDH-mediated lactate conversion could ultimately become a clinically viable metabolic molecular imaging method for measuring disease progression and tumor response to therapy as a complementary addition to anatomic imaging and ${ }^{1} \mathrm{H}$ magnetic resonance spectroscopy (MRS)/magnetic resonance spectroscopic imaging (MRSI). In cardiac research with isolated rat heart and in vivo experiments, others have demonstrated the ability of hyperpolarized ${ }^{13} \mathrm{C}$ to directly evaluate pyruvate

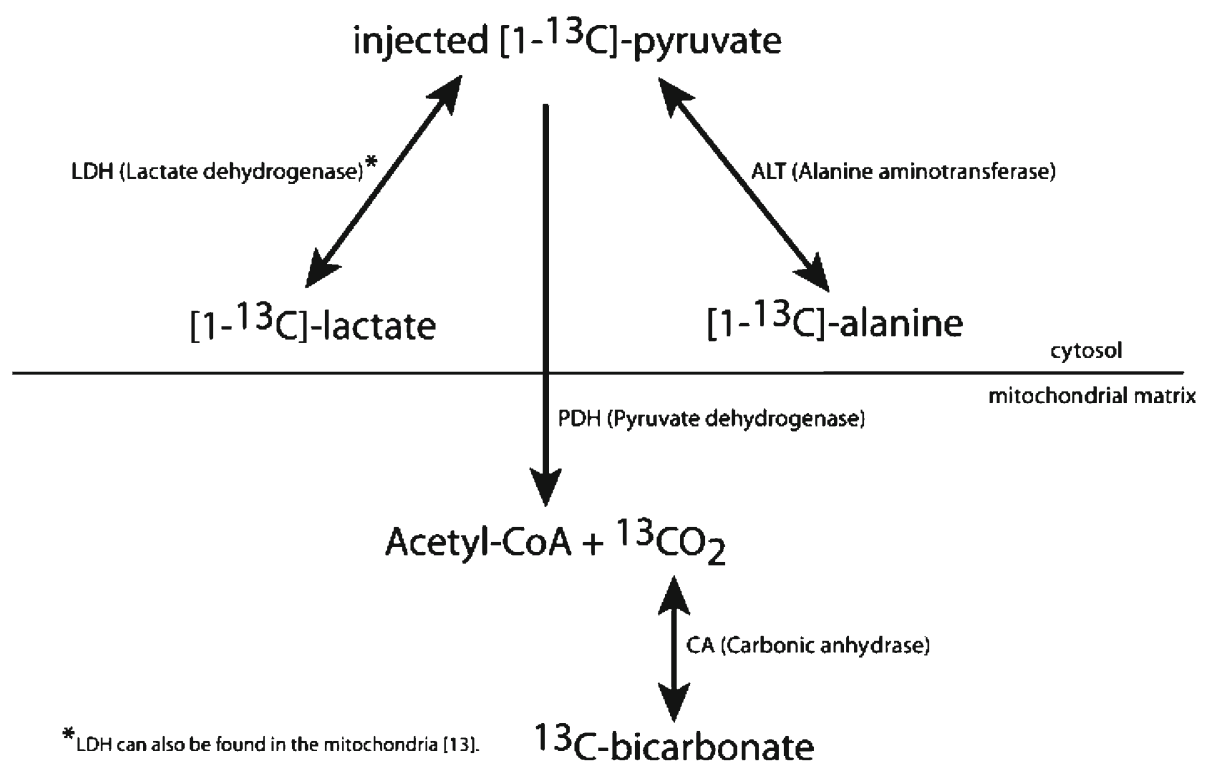

Fig. 1. Diagram of metabolic pathways investigated with DNP hyperpolarized $\left[/-{ }^{13} \mathrm{C}\right]$-pyruvate. Pyruvate/lactate conversion is catalyzed by lactate dehydrogenase $(L D H)$, and pyruvate/alanine conversion is catalyzed by alanine transaminase $(A L T)$. Pyruvate is irreversibly converted to acetyl-CoA and $\mathrm{CO}_{2}$ by pyruvate dehydrogenase $(P D H)$, and $\mathrm{CO}_{2}$ is in a pH-dependent equilibrium with bicarbonate. 
dehydrogenase (PDH) flux by way of bicarbonate conversion [16-17]. PDH flux is a key step in normal and diseased cardiac metabolism, and there has been an initial report of altered bicarbonate levels during ischemia in the pig heart [18] and in a rat model of diabetes [17]. Although all of the above metabolic imaging studies used $\left[1-{ }^{13} \mathrm{C}\right]$ pyruvate, hyperpolarization of other molecular agents is also possible. For example, hyperpolarized $\left[1-{ }^{13} \mathrm{C}\right]$ lactate can be used to examine the LDH back reaction [19]. In addition, hyperpolarized bicarbonate $\left(\mathrm{H}^{13} \mathrm{CO}_{3}-\right)$, which converts to ${ }^{13} \mathrm{CO}_{2}$ in a pH-dependent manner governed by the reaction catalyzed by the enzyme carbonic anhydrase, can be used to image $\mathrm{pH}$ [20], and this has great clinical potential because many disease processes, such as cancer, exhibit abnormal $\mathrm{pH}$ [21]. Hyperpolarization of yet other molecular imaging agents to explore various other metabolic pathways is being actively researched.

The initial research studies applying hyperpolarized ${ }^{13} \mathrm{C}$ metabolic imaging have focused on animal studies of cancer and cardiac metabolism. Thus far, there have been no published reports focusing on the investigation of liver metabolism with hyperpolarized ${ }^{13} \mathrm{C}$ technology. Therefore, the goals of this study were to characterize normal liver metabolism with hyperpolarized ${ }^{13} \mathrm{C}$ slice-localized dynamic spectroscopy and 3D-MRSI and to investigate the ability of this technique to detect a change in metabolic state, specifically fasting. Whereas others have isolated changes based on LDH and PDH activity, in this work, we aimed to focus on detection of alanine levels following injection of hyperpolarized pyruvate to probe ALT activity in the fasted state. Serum ALT levels have been known to change in many liver diseases [22], so therefore ALT-mediated flux could be an additional biomarker of interest for liver disease.

\section{Materials and Methods}

The overall process for the hyperpolarized imaging experiments was as follows: prepare a sample to be polarized, polarize with a DNP polarizer, perform anatomical imaging of the rat while waiting for the polarization to build up, dissolve and eject a liquid sample from the polarizer, inject the sample into a rat inside a clinical magnetic resonance (MR) scanner, and perform either slice dynamic spectroscopy or 3D-MRSI. A total of 20 rat experiments were conducted (five normal dynamic, five fasted dynamic, five normal MRSI, five fasted MRSI).

\section{Polarizer and Preparations}

A HyperSense DNP polarizer (Oxford Instruments, Abingdon, UK) was used in this study. Following previously described methods [4-5], $32 \mu \mathrm{L}$ (about $40 \mathrm{mg}$ ) of $\left[1-{ }^{13} \mathrm{C}\right]$ pyruvic acid (Isotec, Miamisburg, OH, USA) with $15 \mathrm{mM}$ OX63 trityl radical (Oxford Instruments, Abingdon, UK) was polarized in a field of $3.35 \mathrm{~T}$ at approximately $1.4 \mathrm{~K}$ by irradiation with $94.116-\mathrm{GHz}$ microwaves. After approximately $1 \mathrm{~h}$ when the solid-state polarization neared its exponential asymptote, an aqueous solution with $5.96 \mathrm{~g} / \mathrm{L}$ Tris
(40 mM), $4.00 \mathrm{~g} / \mathrm{L} \mathrm{NaOH}(100 \mathrm{mM})$, and $0.1 \mathrm{mg} / \mathrm{L} \mathrm{Na} \mathrm{Na}_{2}$ ethylenediaminetetraacetate acid was injected into the DNP polarizer, heated, and used for rapid thawing and dissolution of the solid-state sample. The amounts of solvent, $\mathrm{NaOH}$, and Tris buffer used were calculated to produce a final polarized sodium pyruvate concentration of $100 \mathrm{mM}$ and a pH of approximately 7.6. After the rapid thawing, the dissolved material was ejected into a flask resting on ice. Immediately thereafter, a small aliquot $(\sim 0.5 \mathrm{~mL})$ of the hyperpolarized $\left[1-{ }^{13} \mathrm{C}\right]$ pyruvate solution was used to measure the level of polarization achieved in solution, and at the same time another $\sim 2.4 \mathrm{~mL}$ was taken to the MR scanner to be injected into a rat over a 12 -s period followed by a normal saline flush.

\section{Animal Handling}

All animal studies were carried out under a protocol approved by the University of California San Francisco Institutional Animal Care and Use Committee. Male Sprague-Dawley rats weighing $\sim 300 \mathrm{~g}$ were either allowed to feed freely on standard rat chow (normal, nonfasted) or had their food removed $24 \mathrm{~h}$ before each hyperpolarized study (fasted). For each experiment, the rat was placed on a heated pad and anesthetized with isoflurane (2-3\%). A catheter was introduced into the tail vein for the eventual intravenous administration of hyperpolarized pyruvate solution, and the rat was transferred to a heated pad in the radio frequency $(\mathrm{RF})$ coil in the MR scanner. While in the scanner, anesthesia was maintained by a continual delivery of isoflurane (1-2\%) via a long tube to a nose cone, with an oxygen flow of $1 \mathrm{~L} / \mathrm{min}$. The rat's vital signs (heart rate and oxygen saturation) were continually monitored. Care was taken to ensure that body temperature was maintained at $37^{\circ} \mathrm{C}$ throughout the imaging procedures by maintaining a flow of heated water through the pad underneath the rat.

\section{MRI, MRS, and MRSI Studies}

All studies were performed using a $3 \mathrm{~T}$ GE Signa ${ }^{\mathrm{TM}}$ scanner (GE Healthcare, Waukesha, WI, USA) equipped with the multinuclear spectroscopy hardware package. The RF coil used in these experiments was a dual-tuned ${ }^{1} \mathrm{H}^{-13} \mathrm{C}$ coil with a quadrature ${ }^{13} \mathrm{C}$ channel and linear ${ }^{1} \mathrm{H}$ channel construction based on an earlier design [23] and used in prior hyperpolarized ${ }^{13} \mathrm{C}$ pyruvate rat imaging studies [10]. The inner coil diameter was $8 \mathrm{~cm}$, and the length of the coil was $9 \mathrm{~cm}$ to accommodate rats of varying size.

T2-weighted anatomical images were obtained in all three planes using a fast spin-echo sequence. Axial and sagittal images were each acquired in approximately $10 \mathrm{~min}$ with a $10-\mathrm{cm}$ field of view (FOV), $192 \times 192$ matrix, 2-mm-thick slices and NEX $=6$. Coronal images were acquired with a 12 -cm FOV, $192 \times 192$ matrix, 1.5-mm-thick slices and NEX $=6$ with a scan time of $10 \mathrm{~min}$. The total imaging time required to obtain images in all three planes was thus approximately $30 \mathrm{~min}$.

For the slice localized liver dynamic MRS experiments, a double spin-echo pulse sequence with a $5^{\circ}$ flip selective RF excitation pulse (15-mm axial slice localization) and a pair of nonlocalized $180^{\circ}$ hyperbolic secant refocusing pulses was used [24]. A TE of $35 \mathrm{~ms}$ (half-echo collected), a repetition time (TR) of $3 \mathrm{~s}$, and a readout filter of $5,000 \mathrm{~Hz} / 2,048 \mathrm{pts}$ were used for these studies. The acquisition localized to a slice through the liver started at the beginning of a 12 -s manual injection of $\left[1-{ }^{13} \mathrm{C}\right]$ pyruvate into the rat tail vein. 

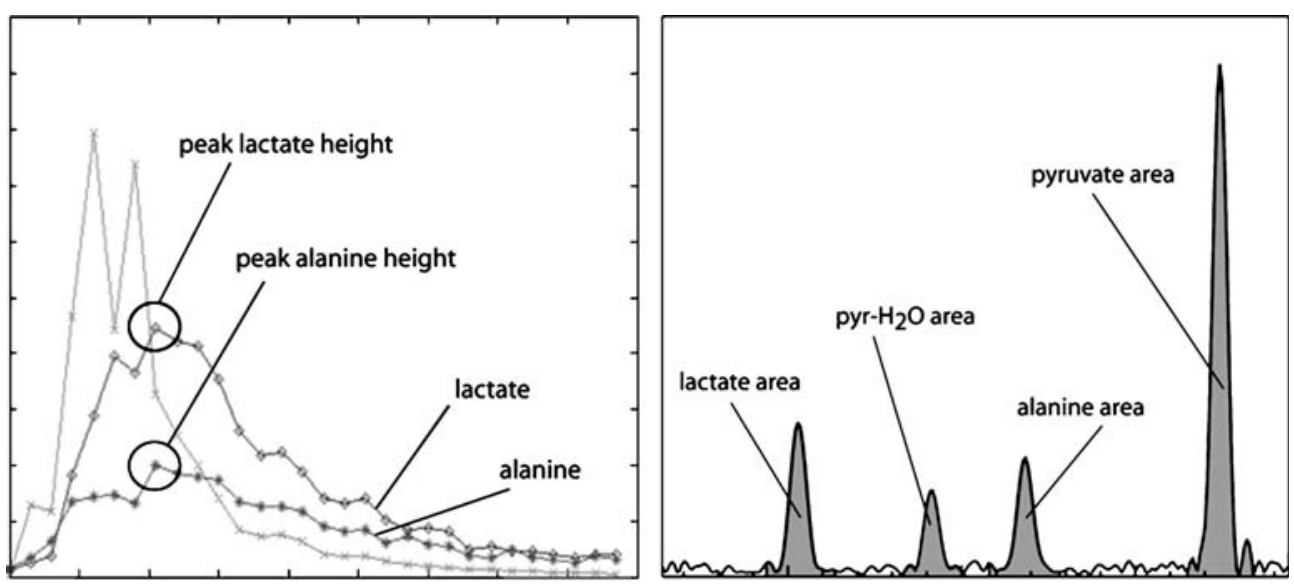

Fig. 2. Example demonstrating the quantification procedures for slice MRS and $3 \mathrm{D}-\mathrm{MRSI} .{ }^{13} \mathrm{C}$-lactate and ${ }^{13} \mathrm{C}$-alanine peak heights were important parameters in the final processed dynamic curves (left). The areas of hyperpolarized ${ }^{13} \mathrm{C}$ resonances in the 3D-MRSI spectra were used in quantitative comparisons (right). The MRS example comes from data plotted in Fig. 3, and the 3D-MRSI example comes from a voxel from Fig. 5.
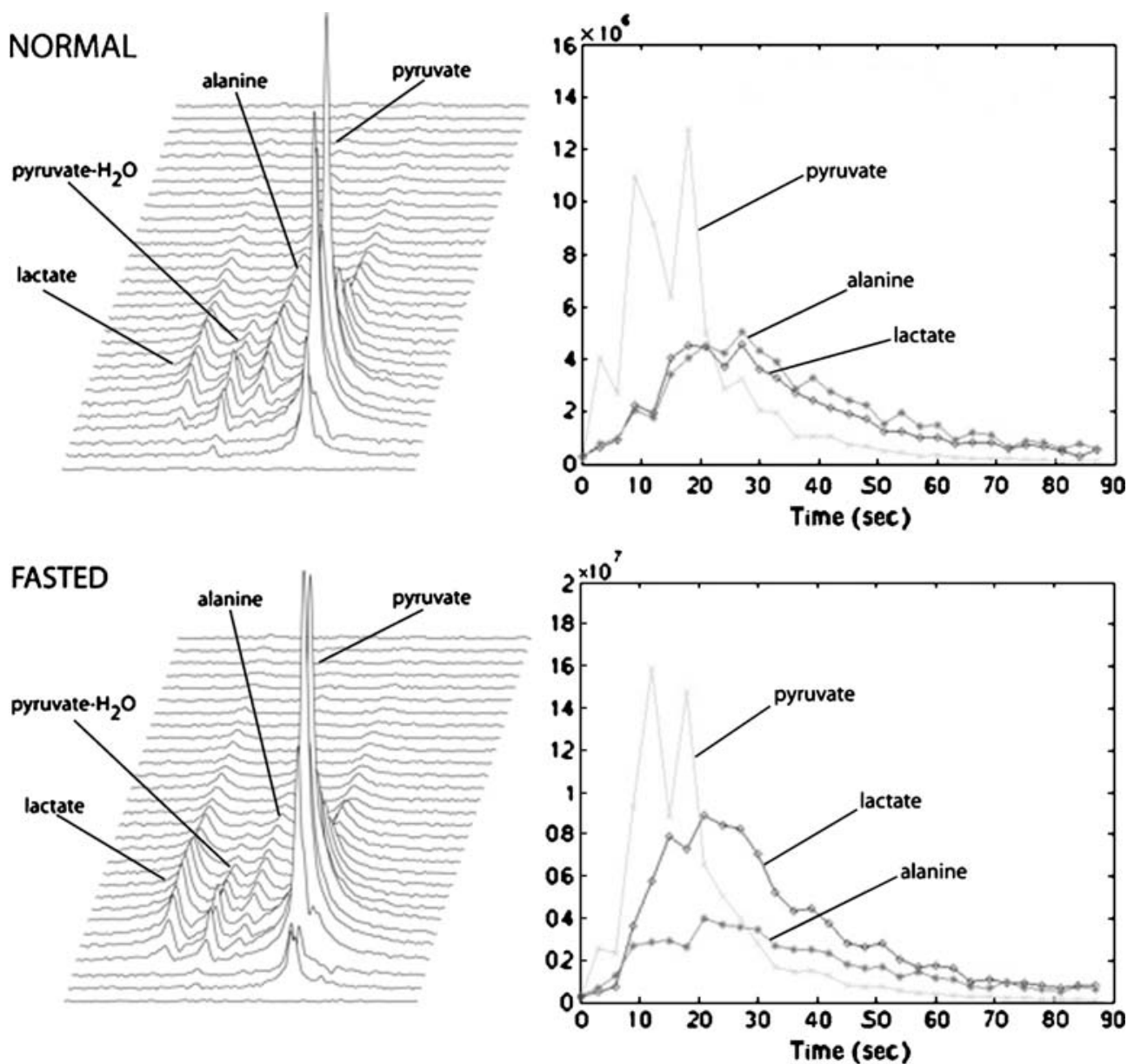

Fig. 3. Comparison of normal (top) and fasted (bottom) rat liver representative dynamic data. The final processed dynamic curves were derived from the stack plots (left). The alanine and lactate levels were similar in the normal rat liver case (top right), but lactate levels were much higher than alanine levels in the fasted rat liver case (bottom right). 
The $3 \mathrm{D}{ }^{13} \mathrm{C}$ MRSI data were acquired in $14 \mathrm{~s}$ (starting $25 \mathrm{~s}$ after the start of injection) with a slab-selective variable small tip angle excitation pulse, double spin-echo refocusing pulses, and a flyback echo-planar readout trajectory $[11,24]$. An $8 \times 8$ phase encoding matrix with a flyback echo-planar trajectory on the $Z$-axis $(8 \times 8 \times 16$ effective matrix) was used with $10 \times 10 \times 10-\mathrm{mm}$ spatial resolution $\left(1.0-\mathrm{cm}^{3}\right.$ voxel resolution) with an $80 \times 80 \times 160-\mathrm{mm}$ FOV to cover the rat torso and abdomen. The flyback echo-planar trajectory was designed for a $581-\mathrm{Hz}$ spectral bandwidth to include ${ }^{13} \mathrm{C}$ lactate, ${ }^{13} \mathrm{C}$ alanine, and ${ }^{13} \mathrm{C}$ pyruvate without spectral aliasing. A total of 59 readout/rewind lobes were included during each readout for a spectral resolution of $9.83 \mathrm{~Hz}$. With a readout filter of $25,000 \mathrm{~Hz} /$ 2,538 points, $16 \mathrm{k}$-space points were acquired during each TR [25]. The TE for the MRSI acquisition was $140 \mathrm{~ms}$ (readout was centered on the center of the second spin-echo, full echo collected), and the TR was $215 \mathrm{~ms}$. As described previously [11], a variable flip angle (VFA) scheme [26], with increasing flip angle over time to compensate for the loss in hyperpolarized signal, was used in the in vivo experiments. Reordering of phase encodes to collect data near the k-space origin first was also employed as previously described [11].

\section{Data Processing}

Fig. 2 shows an example of the dynamic and spectral parameters for data processing in the slice MRS and 3D-MRSI experiments. The MRS example comes from data plotted in Fig. 3, and the 3D-MRSI example comes from a voxel from Fig. 5. Dynamic MRS data, processed with MATLAB ${ }^{\mathrm{TM}}$, were apodized in the time domain with a $10-\mathrm{Hz}$ Lorentzian filter, Fourier-transformed along the time dimension and taken from the resulting magnitude spectra. Magnitude dynamic curves were obtained for pyruvate, pyruvate- $\mathrm{H}_{2} \mathrm{O}$, lactate, and alanine, from which peak lactate to alanine ratios were derived (Fig. 2 left). A nonparametric statistical test (used to avoid any normality assumptions), the Mann-Whitney rank-sum test, was used to compare the peak lactate to alanine ratio between normal and fasted groups. For 3D-MRSI acquisitions, the reconstruction and analysis procedures, described previously in more detail [11, 24], were carried out with specialized custom MRSI software [27] as follows: (1) the raw flyback data were subsampled and ordered to obtain a 4D matrix of kspace data, (2) each FID was apodized and a linear phase correction was applied to the spectral samples as described in [25] to account for the tilted k-space trajectory characteristic of the flyback readout, and (3) a 4D Fourier transform with zero padding of the spectra was performed. From the processed 3D magnitude spectral data, for each rat, the voxels localized to nonvasculature liver tissue were identified from the proton anatomical images and the ${ }^{13} \mathrm{C}$ spectra with sufficient signal-tonoise ratios $(>5)$ were quantified. For each liver voxel, the area under the spectral resonances of pyruvate, pyruvate- $\mathrm{H}_{2} \mathrm{O}$, lactate, and alanine were calculated, with the sum of these four areas defined as the total carbon area (Fig. 2 right). Lactate area to total carbon area and alanine area to total carbon area were calculated for each voxel and then averaged over all selected liver voxels to derive the test statistics average lactate to total carbon ratio and average alanine to total carbon ratio. The Mann-Whitney rank-sum test was used to compare these test statistics between normal and fasted groups.

\section{Results}

\section{Dynamic MRS of Normal and Fasted Rat Liver}

Fig. 3 shows representative dynamic curves from slices localized to the livers of normal and fasted rats. These final dynamic curves were derived from the stack plot insets in which each horizontal line in a stack plot represents a separate magnitude spectrum of the hyperpolarized species collected every $3 \mathrm{~s}$. For easier viewing, the pyruvate curve has been scaled down by a factor of 4 and the nonmetabolically active pyruvate-hydrate, which is in equilibrium with pyruvate, was omitted from the plots in Fig. 3. In the dynamic curves, each marked point represents the intensity of pyruvate $(\sim 171 \mathrm{ppm})$, lactate $(\sim 183 \mathrm{ppm})$, and alanine $(\sim 176 \mathrm{ppm})$ at that time point, i.e., a trace of those ridges in the associated stack plot, showing the uptake and conversion of pyruvate. Typically, the lactate and alanine curves showed a plateau around 20-30 s after injection, meaning the highest lactate and alanine SNR occurred in this range. This is important for picking an imaging window for the 3DMRSI acquisitions, in which the SNR from each voxel is lower than in the slices in the MRS experiments. Qualitatively, the lactate and alanine curves in the normal rats had similar maximum amplitudes while there was a dramatic difference in the fasted rats. Fig. 4 shows the peak lactate to alanine ratios for normal $(1.18 \pm 0.18,[1.27,1.39,1.21,1.09$, $0.91])$ and fasted rats $(1.82 \pm 0.40,[2.28,2.23,1.54,1.42$, 1.64]). Using a Mann-Whitney rank-sum test, there was a statistically significant difference in lactate to alanine ratio $(P<0.01)$. Also, these data demonstrated no overlap between the groups.

\section{D-MRSI of Normal and Fasted Rat Liver}

Fig. 5 shows representative axial slices from 3D-MRSI spectra with $1 \times 1 \times 1-\mathrm{cm}$ voxel resolution of normal and fasted rat liver (typically, the rat liver spans a couple of slices). The proton images serve as anatomical references for the spectra and show vasculature regions in addition to liver parenchyma. All the fasted liver voxel spectra showed a high lactate to alanine ratio. Fig. 6 shows the average lactate to alanine ratios for normal $(1.18 \pm 0.16,[1.42,1.12,1.24,1.11$, $0.99])$ and fasted rats $(1.78 \pm 0.15,[1.85,1.56,1.85,1.94$, 1.65]) in a manner similar to Fig. 4 except with 3D-MRSI data. The Fig. 6 data corroborate the Fig. 4 data, providing further evidence of an effect. In addition, the Fig. 6 data show greater separation between the normal and fasted groups and less spread within the groups, which was probably due to selecting localized liver voxels instead of using tissue from a whole slice for the analysis. Qualitatively in the spectra, the liver lactate levels looked comparable between the normal and fasted states, but alanine seemed lower in the latter state. As described in the "Materials and Methods" section, the average lactate area to total carbon area 


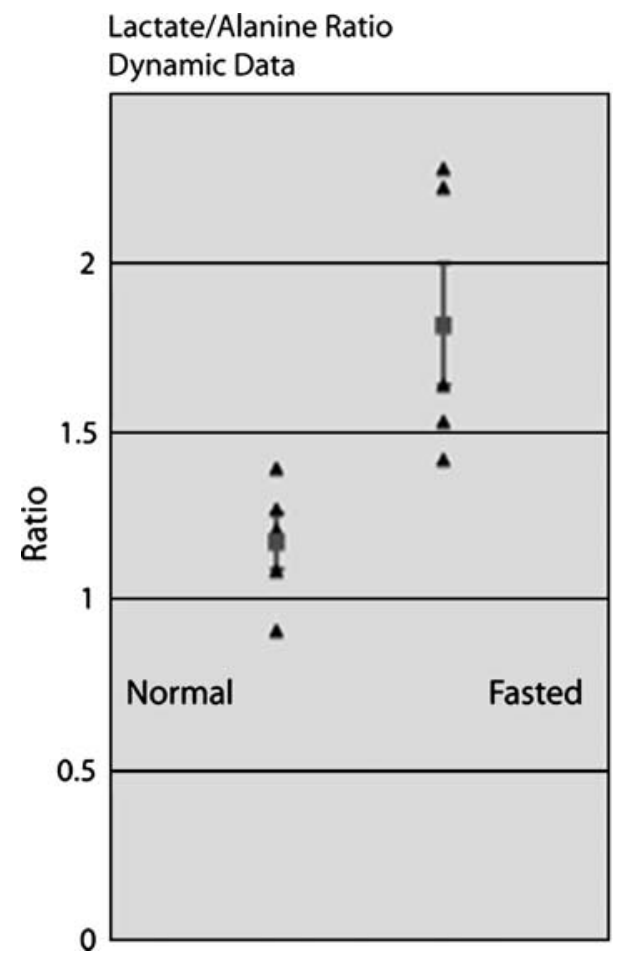

Fig. 4. The data points and mean values for peak lactate to alanine ratios are shown from normal and fasted rat MRS slice acquisitions. Normal rat liver showed a significantly lower lactate to alanine ratio $(P<0.01)$ than fasted rat liver. Mean/SD-(normal 1.18 \pm 0.18 , fasted 1.82 \pm 0.40 ). Note: triangular markers show the collected data points and the square marker/error bars show the mean/standard errors.
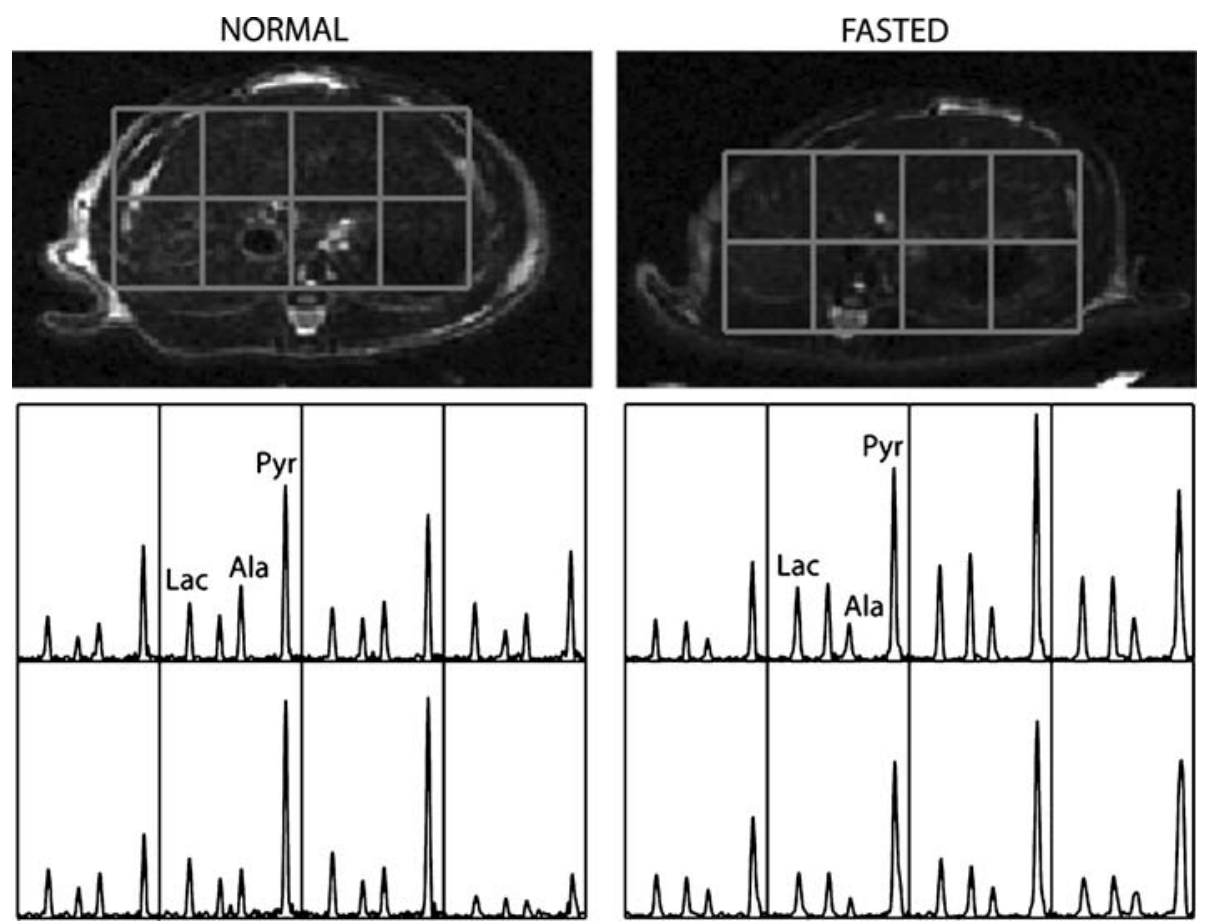

Fig. 5. Comparison of representative liver slice spectra from 3D-MRSI acquisitions on normal (left) and fasted (right) rats. The spectra from normal liver showed similar levels of ${ }^{13} \mathrm{C}$-lactate and ${ }^{13} \mathrm{C}$-alanine. The spectra from fasted liver showed relatively lower levels of ${ }^{13} \mathrm{C}$-alanine. and average alanine area to total carbon area ratios were calculated for each rat. Fig. 7 shows these lactate fractions (normal liver lactate to total carbon $0.22 \pm 0.026$, [0.264, $0.207,0.211,0.200,0.229]$; fasted liver lactate to total carbon $0.23 \pm 0.045,[0.305,0.232,0.191,0.212,0.208])$ and alanine fractions (normal liver alanine to total carbon $0.19 \pm 0.008$, $[0.186,0.185,0.184,0.190,0.202]$; fasted liver alanine to total carbon $0.13 \pm 0.026,[0.165,0.146,0.103,0.110,0.126])$. Using a Mann-Whitney rank-sum test, there was no statistically significant difference in lactate to total carbon area between normal and fasted groups $(P=0.42)$, but there was a statistically significant difference in alanine to total carbon area between normal and fasted groups $(P<0.01)$. With the small sample size and low power, it is hard to say with complete certainty that fasting had no effect on lactate fraction, but the means/overlap in the data were strongly suggestive of lactate fraction being relatively constant for all rats. However, the decrease in alanine was statistically significant and specific, with no overlap between groups.

\section{Discussion}

There have been many recent reports that have shown hyperpolarized ${ }^{13} \mathrm{C} \mathrm{MR}$ to be sensitive to physiological perturbations in enzyme-mediated flux involving $\mathrm{LDH}$ [9, 11-12] and PDH [16-17]. In this work, we have demonstrated that hyperpolarized ${ }^{13} \mathrm{C} \mathrm{MR}$ is also sensitive to physiological perturbations in ALT flux and shown that it is possible to detect changes in metabolism in the liver with this technology. Day et al. [12] demonstrated that metabolic

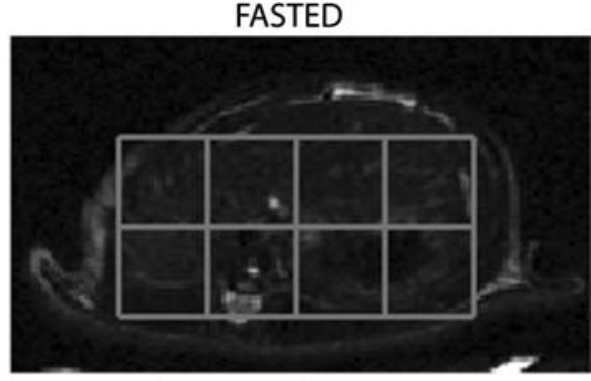




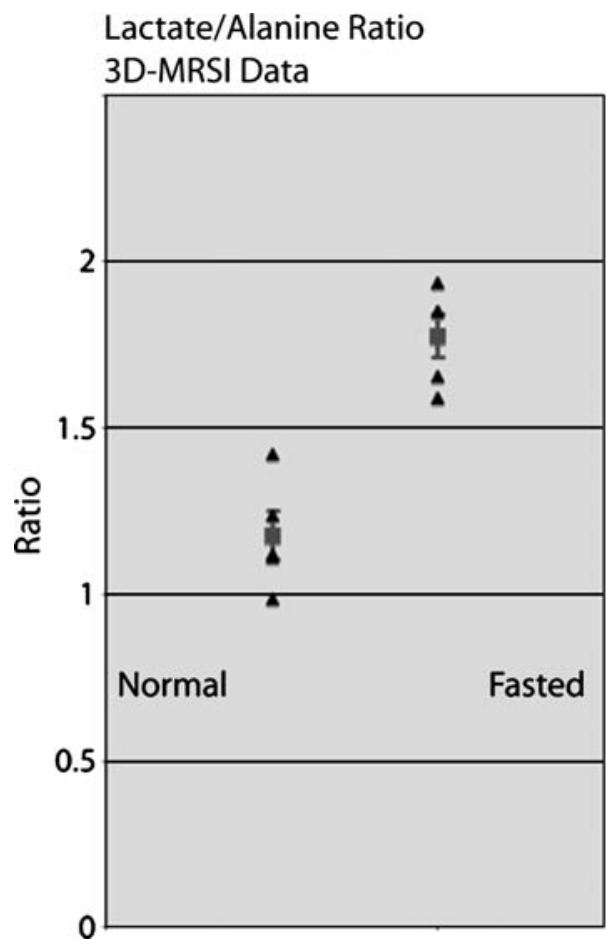

Fig. 6. Lactate area to alanine area from 3D-MRSI studies (averaged over liver voxels per rat) of normal and fasted liver. Normal rat liver showed a significantly lower lactate area to alanine area ratio $(P<0.01)$ than fasted rat liver. These data corroborate the dynamic MRS data, with the means matching closely and indicating the same conclusion. Mean/SD(normal 1.18 \pm 0.16 , fasted $1.78 \pm 0.15$ ). (Note: triangular markers show the collected data points and the square marker/error bars show the mean/standard errors.)

conversion from the injected pyruvate comes from rapid enzyme-mediated exchange of the ${ }^{13} \mathrm{C}$ label, i.e., isotope redistribution to endogenous pools of metabolites [12]. It was specifically shown that the detected lactate spectra reflected the existing pool size of endogenous lactate. This study also demonstrated that, by adding more unlabeled lactate to cells injected with hyperpolarized ${ }^{13} \mathrm{C}$ pyruvate, higher detected ${ }^{13} \mathrm{C}$ lactate was observed due to transfer of label to the unlabeled lactate pool [12]. It was also shown that in vivo there was a decrease in detected lactate when the NADH coenzyme for LDH was inhibited, meaning flux toward lactate and thus transfer of hyperpolarized label to lactate was inhibited [12].
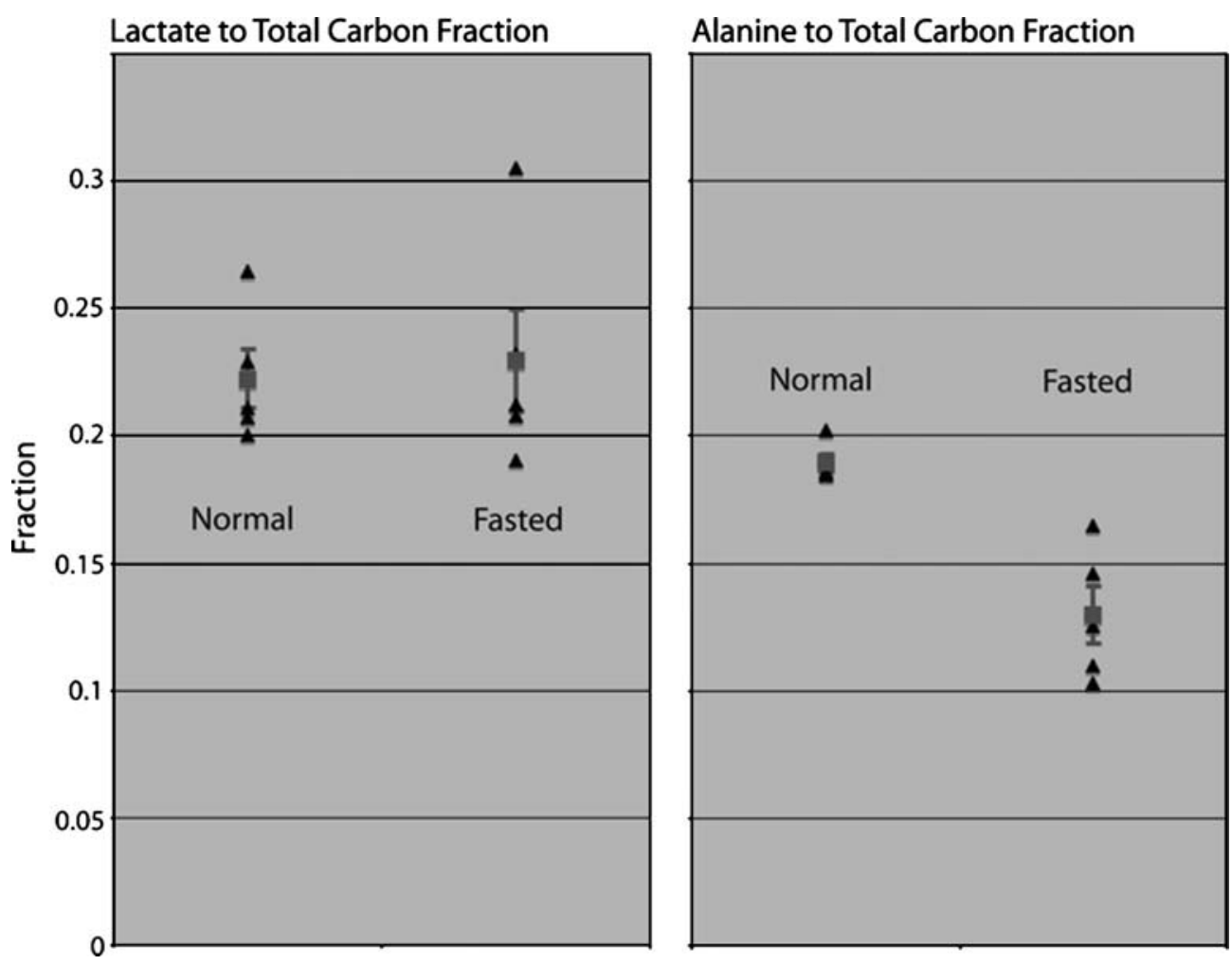

Fig. 7. Lactate area to total carbon-13 fraction (left) and alanine area to total carbon-13 fraction (right) from 3D-MRSI studies (averaged over liver voxels per rat) of normal and fasted liver. The lactate to total carbon ratio was similar between normal and fasted groups, demonstrating that lactate levels were relatively constant. Mean/SD—(normal $0.22 \pm 0.026$, fasted $0.23 \pm 0.045$, $P=0.42$ ). The alanine to total carbon ratio was significantly lower for fasted rat liver. Mean/SD-(normal $0.19 \pm 0.008$, fasted $0.13 \pm 0.026, P<0.01)$. Note: triangular markers show the collected data points and the square marker/error bars show the mean and standard errors. In addition, the five normal alanine to total carbon points overlap, thus obscuring some points. 
While these prior studies focused on the LDH pathway, this study demonstrated that the level of ${ }^{13} \mathrm{C}$ alanine produced in the murine liver from hyperpolarized pyruvate under the influence of ALT flux was sensitive to the physiologic perturbation of fasting. This alteration in alanine levels is supported by prior metabolic studies demonstrating that alanine is the key substrate utilized during gluconeogenesis, e.g., due to fasting [28]. Gluconeogenic alanine conversion during fasting has also been shown in previous biochemical investigations involving perfused rat liver [29-30]. By infusing labeled alanine, Cohen [29] showed that alanine utilization and glucose production increased according to the degree of gluconeogenesis, specifically showing more glucose production in 24-h fasted rats than in 12-h fasted rats and the highest production in drug-induced diabetic rats. In addition, Stromski et al. [31] observed in vivo glucose production from alanine. Thus, the finding that alanine levels changed during fasting has been demonstrated in prior biochemical studies, but what is new in this study is the demonstration that this alteration can be detected in vivo using an MR metabolic imaging technique.

Hyperpolarized technology for the measurement of ${ }^{13} \mathrm{C}$ metabolic flux has many advantages, including high SNR, virtually zero background signal, and large chemical shift dispersion. The use of ${ }^{13} \mathrm{C}$ molecular imaging agents has the additional benefits of no radioactivity and no heavy isotope effects (e.g., deuterium which has twice the molecular weight of water). The method is general in the sense that many metabolic pathways could be probed, especially with the continued development of preparations for the hyperpolarization of agents other than pyruvate [19, 20]. Hyperpolarized spectroscopy with ${ }^{13} \mathrm{C}$ pyruvate can be viewed as a pyruvate challenge experiment employing a non-steady-state measurement after an injection of a pyruvate bolus well exceeding physiological concentrations. Regardless of the supraphysiological levels of the injected substrate, the reactions involving pyruvate have already shown promise in the assessment of glycolysis through LDH [9, 11-12] and cardiac metabolism through PDH $[16,17]$. The pyruvate to lactate pathway is extremely important for cancer applications because of the high lactate flux found in most tumors as dictated by the Warburg principle [15]. For liver disease, flux through the ALT pathway might also have medical relevance. Clinical liver function tests routinely use measurements of ALT levels in the blood, with high levels correlating with disease. For example, high serum ALT levels (i.e., twofold to tenfold normal) can indicate hepatitis, fatty liver, and hepatotoxicity [22]. The presumed mechanism responsible for elevated levels of ALT is injury to hepatocytes and the consequent leakage of ALT into the bloodstream [22], making ALT levels dependent on the degree of liver injury. This does not necessarily represent a change in ALT concentrations in liver cells, but if there is indeed altered ALT and alanine concentrations in the liver resulting in altered flux of the ${ }^{13} \mathrm{C}$ label to alanine, then anatomically localized ${ }^{13} \mathrm{C}$ metabolic imaging with hyper- polarized pyruvate as the substrate could be a valuable technique to probe these changes. Using a hyperpolarized molecular probe to monitor changes in localized ${ }^{13} \mathrm{C}$ alanine distribution could potentially be a more specific assay than a serum ALT test, which can be complicated by high ALT levels occurring from nonhepatic causes [32]. Additionally, using hyperpolarized $\left[1-{ }^{13} \mathrm{C}\right]$ pyruvate to monitor ALT flux may be a useful biomarker of disease progression and response to therapy. The significant in vivo change in liver metabolism during fasting observed in this study is an exciting initial demonstration of the potential value for liver hyperpolarized MR metabolic imaging, as well as an encouraging result for future liver disease studies using hyperpolarized MR.

\section{Conclusions}

Normal and fasted rat liver were characterized with hyperpolarized ${ }^{13} \mathrm{C}$ slice-localized dynamic magnetic resonance spectroscopy and 3D magnetic resonance spectroscopic imaging. Fasted rat liver showed significantly lower levels of alanine in both the dynamic MRS and 3D-MRSI data, demonstrating the feasibility of hyperpolarized MR technology for detecting a change in metabolic state in the liver.

Acknowledgements. We would like to thank Dr. James Tropp for designing and building the animal coil used in this study, Dr. Jeffrey MacDonald for discussions on metabolism, and Srivathsa Veeraraghavan and Vickie Zhang for help during the in vivo experiments. This study was supported by NIH grant R01 EB007588 and UC Discovery grant ITL-BIO04-10148, in conjunction with GE Healthcare.

Open Access. This article is distributed under the terms of the Creative Commons Attribution Noncommercial License which permits any noncommercial use, distribution, and reproduction in any medium, provided the original author(s) and source are credited.

\section{References}

1. Gruetter R, Adriany G, Choi I-Y et al (2003) Localized in vivo ${ }^{13} \mathrm{C}$ NMR spectroscopy of the brain. NMR Biomed 16:313-338

2. de Graaf RA, Mason GF, Patel AB et al (2003) In vivo ${ }^{1} \mathrm{H}-\left[{ }^{13} \mathrm{C}\right]-\mathrm{NMR}$ spectroscopy of cerebral metabolism. NMR Biomed 16:339-357

3. Ross B, Lin A, Harris K et al (2003) Clinical experience with ${ }^{13} \mathrm{MRS}$ in vivo. NMR Biomed 16:358-369

4. Ardenkjaer-Larson JH, Fridlund B, Gram A et al (2003) Increase in signal-to-noise ration of $>10,000$ times in liquid-state NMR. Proc Natl Acad Sci USA 100:10158-10163

5. Wolber J, Ellner F, Fridlund B et al (2004) Generating highly polarized nuclear spins in solution using dynamic nuclear polarization. Nucl Instr Meth Phys Res A 526:173-181

6. Golman K, Ardenkjaer-Larsen JH, Peterson JS et al (2003) Molecular imaging with endogenous substances. Proc Natl Acad Sci USA 100:10435-10439

7. Johannson E, Mansson S, Wirestam R et al (2004) Cerebral perfusion assessment by bolus tracking using hyperpolarized ${ }^{13} \mathrm{C}$. Magn Reson Med 51:464-472

8. Golman K, Zandt R, Thaning M (2006) Real-time metabolic imaging. Proc Natl Acad Sci USA 103:11270-11275

9. Golman K, Zandt RI, Lerche M et al (2006) Metabolic imaging by hyperpolarized ${ }^{13} \mathrm{C}$ magnetic resonance imaging for in vivo tumor diagnosis. Cancer Res 66:10855-10860

10. Kohler SJ, Yen Y, Wolber J et al (2007) In vivo ${ }^{13}$ carbon metabolic imaging at $3 \mathrm{~T}$ with hyperpolarized ${ }^{13} \mathrm{C}-1$-pyruvate. Magn Reson Med 58:65-69 
11. Chen AP, Albers MJ, Cunningham CH et al (2007) Hyperpolarized C13 spectroscopic imaging of the TRAMP mouse at 3T-initial experience. Magn Reson Med 58:1099-1106

12. Day SE, Kettunen MI, Gallagher FA et al (2007) Detecting tumor response to treatment using hyperpolarized ${ }^{13} \mathrm{C}$ magnetic resonance imaging and spectroscopy. Nat Med 13:1382-1387, Erratum 13:1521

13. Brooks GA, Dubouchaud H, Brown M et al (1999) Role of mitochondrial lactate dehydrogenase and lactate oxidation in the intracellular lactate shuttle. Proc Natl Acad Sci USA 96:1129-1134

14. Board M, Humm S, Newsholme E (1990) Maximum activities of key enzymes of glycolysis, glutaminolysis, pentose phosphate pathway and tricarboxylic acid cycle in normal, neoplastic and suppressed cells. Biochem J 265:503-509

15. DeBerardinis RJ, Lum JJ, Hatzivassiliou G et al (2008) The biology of cancer: metabolic reprogramming fuels cell growth and proliferation. Cell Metab 7:11-20

16. Merritt ME, Harrison C, Storey C et al (2007) Hyperpolarized ${ }^{13} \mathrm{C}$ allows a direct measure of flux through a single enzyme-catalyzed step by NMR. Proc Natl Acad Sci USA 104:19773-19777

17. Schroeder M, Cochlin L, Heather L et al (2008) In vivo assessment of pyruvate dehydrogenase flux in the heart using hyperpolarized carbon-13 magnetic resonance. Proc Natl Acad Sci USA 105:1205112056

18. Golman K, Peterson JS (2006) Metabolic imaging and other applications of hyperpolarized ${ }^{13} \mathrm{C}$. Acad Radiol 13:932-942

19. Chen AP, Kurhanewicz J, Bok R et al (2008) Feasibility of using hyperpolarized $\left[1-{ }^{13} \mathrm{C}\right]$ lactate as a substrate for in vivo metabolic ${ }^{13} \mathrm{C}$ MRSI studies. Magn Reson Imag 26:721-726

20. Gallagher FA, Kettunen MI, Day SE et al (2008) Magnetic resonance imaging of $\mathrm{pH}$ in vivo using hyperpolarized ${ }^{13} \mathrm{C}$-labelled bicarbonate. Nature 453:940-944
21. Gillies RJ, Raghunand N, Garcia-Martin M et al (2004) pH imaging: a review of $\mathrm{pH}$ measurement methods and applications in cancers. IEEE Eng Med Biol Mag 23:57-64

22. Reddy KR, Faust T (eds) (2006) The clinician's guide to liver disease, 1 st edn. SLACK Incorporated, Thorofare

23. Derby K, Tropp J, Hawryszko C (1990) Design and evaluation of a novel dual-tuned resonator for spectroscopic imaging. J Mag Reson 86:645-651

24. Cunningham CH, Chen AP, Albers MJ et al (2007) Double spin-echo sequence for rapid spectroscopic imaging of hyperpolarized ${ }^{13} \mathrm{C}$. J Magn Reson 187:357-362

25. Cunningham CH, Vigneron DB, Chen AP et al (2005) Design of flyback echo-planar readout gradients for magnetic resonance spectroscopic imaging. Mag Reson Med 54:1286-1289

26. Zhao L, Mulkern R, Tseng C-H et al (1996) Gradient-echo imaging considerations for hyperpolarized ${ }^{129}$ Xe MR. J Magn Reson Series B 113:179-183

27. Nelson SJ (2001) Analysis of volume MRI and MR spectroscopic imaging data for the evaluation of patients with brain tumors. Mag Reson Med 46:228-239

28. Felig P (1973) The glucose-alanine cycle. Metab 22:179-207

29. Cohen SM (1987) ${ }^{13} \mathrm{C}$ and ${ }^{13} \mathrm{P}$ NMR study of gluconeogenesis: utilization of ${ }^{13} \mathrm{C}$-labeled substrates by perfused liver from streptozotocin-diabetic and untreated rats. Biochem 26:563-572

30. Cohen SM (1987) ${ }^{13}$ C NMR study of effects of fasting and diabetes on the metabolism of pyruvate in the tricarboxylic acid cycle and of the utilization of pyruvate and ethanol in lipogenesis in perfused rat liver. Biochem 26:581-589

31. Stromski ME, Arias-Mendoza F, Alger JR et al (1986) Hepatic gluconeogenesis from alanine: ${ }^{13} \mathrm{C}$ nuclear magnetic resonance methodology for in vivo studies. Magn Reson Med 3:24-32

32. Giboney PT (2005) Mildly elevated liver transaminase levels in the asymptomatic patient. Am Fam Physician 71:1105-1110 